\title{
Frequency of and Factors Associated With Severe Maternal Morbidity
}

\author{
William A. Grobman, M.D., M.B.A., Jennifer L. Bailit, M.D., M.P.H., Madeline Murguia Rice, \\ Ph.D., Ronald J. Wapner, M.D., Uma M. Reddy, M.D., M.P.H., Michael W. Varner, M.D., John \\ M. Thorp Jr., M.D., Kenneth J. Leveno, M.D., Steve N. Caritis, M.D., Jay D. lams, M.D., Alan \\ T. Tita, M.D., Ph.D., George Saade, M.D., Yoram Sorokin, M.D., Dwight J. Rouse, M.D., Sean \\ C. Blackwell, M.D., Jorge E. Tolosa, M.D., M.S.C.E., and J. Peter Van Dorsten, M.D. for the \\ Eunice Kennedy Shriver National Institute of Child Health and Human Development \\ (NICHD) Maternal-Fetal Medicine Units (MFMU) Network ${ }^{\star}$ \\ Departments of Obstetrics and Gynecology, Northwestern University, Chicago, IL (W.A.G.); Case \\ Western Reserve University-MetroHealth Medical Center, Cleveland, OH (J.L.B.); Columbia \\ University, New York, NY (R.J.W.); University of Utah Health Sciences Center, Salt Lake City, UT \\ (M.W.V.); University of North Carolina at Chapel Hill, Chapel Hill, NC (J.M.T.); University of Texas \\ Southwestern Medical Center, Dallas, TX (K.J.L.); University of Pittsburgh, Pittsburgh, PA \\ (S.N.C.); The Ohio State University, Columbus, OH (J.D.I.); University of Alabama at \\ Birmingham, Birmingham, AL (A.T. T.); University of Texas Medical Branch, Galveston, TX \\ (G.S.); Wayne State University, Detroit, MI (Y.S.); Brown University, Providence, RI (D.J.R.); \\ University of Texas Health Science Center at Houston-Children's Memorial Hermann Hospital, \\ Houston, TX (S.C.B.); Oregon Health \& Science University, Portland, OR (J.E.T.); Medical \\ University of South Carolina, Charleston, SC (J.P.V.D.); and the George Washington University \\ Biostatistics Center, Washington, DC (M.M.R.); and the Eunice Kennedy Shriver National Institute \\ of Child Health and Human Development, Bethesda, MD (U.M.R.)
}

\section{Abstract}

Objective-To estimate the frequency of severe maternal morbidity, assess its underlying etiologies, and develop a scoring system to predict its occurrence.

\begin{abstract}
Methods-This was a secondary analysis of a National Institute of Child Health and Human Development Maternal-Fetal Medicine Units Network cohort of 115,502 women and their neonates born in 25 hospitals across the United States over a 3-year period. Women were classified as having severe maternal morbidity according to a scoring system that takes into account the occurrence of red blood cell transfusion (> 3 units), intubation, unanticipated surgical intervention, organ failure, and intensive care unit admission. The frequency of severe maternal morbidity was calculated and the underlying etiologies determined. Multivariable analysis identified patient factors present on admission that were independently associated with severe maternal morbidity, these were used to develop a prediction model for severe maternal morbidity.
\end{abstract}

Corresponding author: William A. Grobman, MD, MBA, Department of Obstetrics and Gynecology, Feinberg School of Medicine, Northwestern University, 250 East Superior Street, Suite 05-2175, Chicago, IL 60611,w-grobman@northwestern.edu.

*For a list of other members of the NICHD MFMU, see the Appendix online at http://links.lww.com/xxx. 
Results-Among 115,502 women who delivered during the study period, 332 (2.9 per thousand births, 95\% CI 2.6 - 3.2) experienced severe maternal morbidity. Postpartum hemorrhage was responsible for approximately half of severe maternal morbidity. Multiple patient factors were found to be independently associated with severe maternal morbidity and were used to develop a predictive model with an area under the receiver-operating characteristic curve of 0.80 .

Conclusion-Severe maternal morbidity occurs in approximately 2.9 per 1000 births, is most commonly due to postpartum hemorrhage, and occurs more commonly in association with several identifiable patient characteristics.

After years of decline, the frequency of maternal mortality in the United States has begun to increase. ${ }^{1}$ Consequently, several professional organizations devoted to maternal health held a meeting in 2012 to determine methods to help improve maternal outcomes. ${ }^{2}$ One concern was the lack of a standardized metric to identify women who experience significant maternal morbidity. This absence hinders both: (1) investigation into etiologies of the events that result in severe morbidity, and (2) evidence-based quality improvement initiatives. Correspondingly, the group called for research that would help to develop a standardized measure of severe maternal morbidity that could be used as the foundation for surveillance and continuous quality improvement.

The development of such a measure is a goal that has been shared by others. Geller et al ${ }^{3}$ identified those with very severe morbidity by validating an arithmetical scoring system that took into account whether a woman had an unanticipated surgical intervention, was intubated for more than 12 hours postpartum, received a transfusion more than 3 units, was admitted to the intensive care unit (ICU), or experienced organ failure. However, this measure only has been explored in two studies, both of which were performed in single centers with relatively few patients analyzed. ${ }^{4,5}$

The purpose of the present study was to estimate the frequency of severe maternal morbidity in a large multi-center obstetric population using the system of Geller et al, ${ }^{4}$ and to identify its underlying etiologies, associated factors, and whether a scoring system could be developed to predict its occurrence.

\section{Methods}

Between 2008 and 2011, investigators at 25 medical centers, belonging to the Eunice Kennedy Shriver National Institute of Child Health and Human Development Maternal-Fetal Medicine Units Network, assembled an obstetric cohort in which detailed information was collected by trained and certified nurses on patient characteristics, intrapartum events, pregnancy outcomes, and postpartum courses of women who delivered at their institutions. Full details of the study design and technique of data collection for the Assessment of Perinatal EXcellence (APEX) cohort have been described previously. ${ }^{6}$ Institutional review board approval was obtained at all centers under a waiver of informed consent.

The present analysis included all women in the APEX cohort. Women were given a score according to the classification system developed by Geller et al. ${ }^{4}$ This system assigns points according to whether a parturient had an unanticipated surgical intervention (1 point), was 
intubated for more than 12 hours ( 2 points), received a red blood cell transfusion of greater than 3 units ( 3 points), was admitted to the intensive care unit ( 4 points), or had failure of at least one organ system (5 points). Points are then summated, and those women with 8 or more points are classified as having had severe morbidity. For the present analysis, organ failure was defined as any one of the following: cardiopulmonary arrest, cardiac dysfunction (e.g., cardiomyopathy), adult respiratory distress syndrome (ARDS), pulmonary edema, disseminated intravascular coagulation (DIC), transfusion of platelets, stroke, subdural hematoma, postpartum serum creatinine $>2.0$ or postpartum serum AST/SGOT $>1000$. Also, women were considered to fulfill the intubation criterion if they were intubated on at least one day, given that data for length of intubation were collected in day, not hour, increments.

Women who experienced severe morbidity were identified and the overall frequency in the cohort was determined. Their abstracted data were further reviewed by one of the investigators (WAG) to determine the primary etiology of their morbid outcome. Etiologic categories included: hemorrhage, infection, hypertensive disorders of pregnancy, nonhypertensive and non-infectious cardiopulmonary complications, trauma, iatrogenic complications, and pre-existing maternal medical conditions.

We then investigated which patient factors were independently associated with severe morbidity using multivariable logistic regression to generate odds ratios and $95 \%$ confidence intervals. Model selection and internal validation was performed using k-fold crossvalidation in which the cohort was randomly divided into 10 equal parts and logistic regression models, using backward selection, were generated using every possible combination of 9 of the 10 sets. $^{7}$ Variables with $P<.05$ were retained, and each of the 10 sub-samples was used for validation. A risk score was developed using methods similar to Rassi et al. ${ }^{8}$ Each coefficient from the final multivariable model was divided by the smallest coefficient and rounded to the nearest integer. The area under the receiver-operating characteristic curve (AUC), sensitivity, specificity, and positive and negative likelihood ratios (with 95\% confidence intervals) were then calculated. All tests were two-tailed and $P$ $<.05$ was used to define statistical significance. No imputation for missing data was performed. Analyses were performed using SAS software (SAS Institute, Cary, NC).

\section{Results}

During the study period, 115,502 women delivered. After excluding 7 maternal deaths, 115,495 were included in this analysis. Of the $115,495,0.5 \%(\mathrm{~N}=619)$ had an unanticipated surgical intervention, $0.1 \%(\mathrm{~N}=136)$ were intubated on at least one day, $0.3 \%(\mathrm{~N}=323)$ received a blood transfusion of at least 3 units, $0.6 \%(\mathrm{~N}=735)$ were admitted to the intensive care unit, and $0.6 \%(\mathrm{~N}=709)$ had at least one organ system fail. The summative point totals, as determined by the system of Geller et al, ${ }^{4}$ are provided in Table 1 . Three hundred and thirty-two women (2.9 per thousand births, 95\% CI 2.6 - 3.2) had a point total of 8 or more and thus were classified as having had severe morbidity.

Postpartum hemorrhage was responsible for almost half of severe morbidity $(\mathrm{n}=158$, 47.6\%). Hypertensive complications $(n=68,20.5 \%)$, acute cardiopulmonary complications 
(cardiomyopathy, arrest, acute respiratory distress syndrome, pulmonary edema) $(\mathrm{n}=63$, $19.0 \%)$, infection ( $\mathrm{n}=20,6.0 \%)$, pre-existing maternal medical conditions $(\mathrm{n}=8,2.4 \%)$, venous thromboembolism $(n=4,1.2 \%)$, trauma $(n=4,1.2 \%)$, acute neurologic complications ( $\mathrm{n}=3,0.9 \%)$, iatrogenic events (anaphylaxis, surgical visceral injury) $(\mathrm{n}=2$, $0.6 \%)$, and pregnancy-specific conditions (acute fatty liver, amniotic fluid embolism) $(\mathrm{n}=2$, $0.6 \%$ ) accounted for the remainder.

Patient characteristics, stratified by the occurrence of severe morbidity, are presented in Table 2. Patient factors significantly and independently associated with severe morbidity, along with odds ratios and 95\% confidence intervals, are presented in Table 3. The presence of placenta accreta was the characteristic most strongly associated with severe morbidity, although multiple other associated factors, such as preterm delivery, antenatal anticoagulant or cigarette use, hypertension, diabetes mellitus, abruptio placentae, and prior cesarean delivery also were identified.

The number of points that each characteristic contributed to the risk score are presented in Table 3. Point totals ranged from 0 to 24 , with higher point totals indicating greater frequency of severe maternal morbidity (Table 4). The AUC for the risk score model was 0.80 (95\%CI, 0.77-0.83). Sensitivity, specificity, and likelihood ratios for the various risk score cutoff points are also presented (Table 4).

\section{Discussion}

In this study, we have used the scoring system developed by Geller et al. ${ }^{4}$ to characterize the frequency and factors associated with severe morbidity in a 25 -hospital consortium. ${ }^{6}$ Our results demonstrate that, between 2008 and 2011, severe morbidity occurred in 2.9 per thousand woman who gave birth. Postpartum hemorrhage and hypertensive disorders of pregnancies together accounted for more than two-thirds of the primary underlying causes of severe morbidity. Additionally, the probability of experiencing severe morbidity was related to multiple well-defined patient characteristics.

The observed frequency is slightly lower than that estimated in other reports from developed countries. Wen et al, ${ }^{9}$ using Canadian administrative data, found that severe maternal morbidity occurred in $0.44 \%$ of deliveries. Zwart et al, ${ }^{10}$ analyzing data from a prospective study between 2004 and 2006, estimated that $0.71 \%$ of women giving birth experienced severe maternal morbidity in the Netherlands. Callaghan et a ${ }^{11}$ studied the National Inpatient Sample to estimate severe maternal morbidity among American women and derived an estimate of $1.5 \%$ for the years 2008-2009.

These previous studies differ from the current analysis in several important aspects. Both the Canadian and American estimates were derived from administrative data sets, in which the codes are both limited in their scope, entered primarily for billing, and as Callaghan et al note, subject to errors of omission and commission. ${ }^{9,11}$ Also, the definition of severe morbidity in all of these studies was based on the occurrence of a pre-defined event, such as eclampsia or blood transfusion. ${ }^{9-11}$ However, determining morbidity through such a system could miss women who had severe morbidity because of other diagnoses or causes. These 
approaches also may be too sensitive; even though it is likely that a woman who has received a blood transfusion has experienced some morbidity, it may not have been severe.

Our analysis, in contrast, estimated the frequency of severe morbidity from a dataset that was derived from direct chart abstraction and was specifically constructed for research purposes. We used a system that was developed and validated specifically for the purpose of identifying women with the gravest obstetric morbidity. This system is not dependent on any single factor or diagnosis for identification, but on a constellation of adverse outcomes that could be the end result of any serious untoward or precipitating event.

The use of such a system allows insight into the etiologies that are contributing to severe morbidity. Such knowledge is important from a public health perspective in terms of knowing where to focus prevention and treatment efforts. Our data support the concept that postpartum hemorrhage and hypertensive disease of pregnancy are responsible for a large portion of severe maternal morbidity, and underscore that the continued pursuit of bestpractice care for these conditions may also yield substantial benefits in terms of decreasing the most severe maternal outcomes. ${ }^{12,13}$

Our data also allow insight into the patient factors that are associated with severe morbidity. These factors, as well as the risk score derived from them, could be used to help quantify the level of risk a woman has for a severely morbid outcome. It should be noted, however, that this quantification does not allow precise identification of women who will and will not have severe maternal morbidity. Because of the very low prevalence of severe morbidity, as indicated by Table 4, even with a prediction system with an ROC of 0.80 , a cutoff point that identifies women with a high risk of severe morbidity will simultaneously miss identifying most women who experience severe morbidity. For example, although a score of at least 19 results in a positive predictive value for severe maternal morbidity of $39 \%$, this cutoff will not identify $93 \%$ of women who will experience severe morbidity. Conversely, a cutoff of 5 will result in $67 \%$ of women destined to have severe maternal morbidity being identified, but a positive predictive value of only $0.93 \%$. Thus, it is not evident that this prediction model could be used to effectively identify and change the clinical care of individual women.

Nevertheless, as maternal morbidity and mortality increase in frequency in the United States, the need to better understand their risks and causes increases in importance. Without the ability to measure and analyze these outcomes, the potential for systems improvement is compromised. ${ }^{2}$ Severe morbidity as defined in this analysis, was approximately 50 times as frequent as maternal mortality in our population and could be used more readily to track outcomes and to be incorporated into quality improvement initiatives. Our analysis illustrates the importance of gaining a national consensus on a standard definition of severe maternal morbidity in order to allow standardized surveillance.

\section{Supplementary Material}

Refer to Web version on PubMed Central for supplementary material. 


\section{Acknowledgments}

The project described was supported by grants from the Eunice Kennedy Shriver National Institute of Child Health and Human Development (NICHD) [HD21410, HD27869, HD27915, HD27917, HD34116, HD34208, HD36801, HD40500, HD40512, HD40544, HD40545, HD40560, HD40485, HD53097, HD53118] and the National Center for Research Resources [UL1 RR024989; 5UL1 RR025764] and its contents do not necessarily represent the official views of the NICHD, NCRR, or NIH.

The authors thank the following subcommittee members: Cynthia Milluzzi, R.N., and Joan Moss, R.N.C., M.S.N., who participated in protocol development and coordination between clinical research centers; Elizabeth Thom, Ph.D., and Yuan Zhao, M.S., for protocol/data management and statistical analysis; and Catherine Y. Spong, M.D., and Brian M. Mercer, M.D, for protocol development and oversight.

\section{References}

1. Hogan MC, Foreman KJ, Naghavi M, et al. Maternal mortality for 181 countries, 1980-2008: a systematic analysis of progress towards Millennium Development Goal 5. Lancet. 2010; 375(9726): 1609-23. [PubMed: 20382417]

2. D'Alton M, Bonanno CA, Berkowitz RL, et al. Putting the "M" back in maternal-fetal medicine. Am J Obstet Gynecol. 2013; 208(6):442-8. [PubMed: 23211544]

3. Geller SE, Rosenberg D, Cox SM, Kilpatrick S. Defining a conceptual framework for near-miss maternal morbidity. J Am Med Womens Assoc. 2002; 57(3):135-9. [PubMed: 12146602]

4. Geller SE, Rosenberg D, Cox S, Brown M, Simonson L, Kilpatrick S. A scoring system identified near-miss maternal morbidity during pregnancy. J Clin Epidemiol. 2004; 57(7):716-20. [PubMed: 15358399]

5. You WB, Chandrasekaran S, Sullivan J, Grobman W. Validation of a scoring system to identify women with near-miss maternal morbidity. Am J Perinatol. 2013; 30(1):21-4. [PubMed: 22814799]

6. Bailit JL, Grobman WA, Rice MM, et al. Risk-adjusted models for adverse obstetric outcomes and variation in risk-adjusted outcomes across hospitals. Am J Obstet Gynecol. 2013; 209(5):446, e130. [PubMed: 23891630]

7. Kohavi, R. A study of cross-validation and bootstrap for accuracy estimation and model selection. In: Mellish, CS., editor. IJCAI'95 Proceedings of the 14th international joint conference on artificial intelligence. Vol. 2. San Francisco, CA: Morgan Kaufmann Publishers Inc; 1995.

8. Rassi A Jr, Rassi A, Little WC, et al. Development and validation of a risk score for predicting death in Chagas' heart disease. N Engl J Med. 2006; 355(8):799-808. [PubMed: 16928995]

9. Wen SW, Huang L, Liston R, et al. Severe maternal morbidity in Canada, 1991-2001. CMAJ. 2005; 173(7):759-64. [PubMed: 16186582]

10. Zwart JJ, Richters JM, Ory F, de Vries JI, Bloemenkamp KW, van Roosmalen J. Severe maternal morbidity during pregnancy, delivery and puerperium in the Netherlands: a nationwide populationbased study of 371,000 pregnancies. BJOG. 2008; 115(7):842-50. [PubMed: 18485162]

11. Callaghan WM, Creanga AA, Kuklina EV. Severe Maternal morbidity among delivery and postpartum hospitalizations in the United States. Obstet Gynecol. 2012; 120(5):1029-36. [PubMed: 23090519]

12. Knight M, Callaghan WM, Berg C, et al. Trends in postpartum hemorrhage in high resource countries: a review and recommendations from the International Postpartum Hemorrhage Collaborative Group. BMC Pregnancy Childbirth. 2009; 9:55. [PubMed: 19943928]

13. Menzies J, Magee LA, Li J, et al. Instituting surveillance guidelines and adverse outcomes in preeclampsia. Obstet Gynecol. 2007; 110(1):121-7. [PubMed: 17601906] 


\section{Table 2}

Patient Characteristics by Severe Maternal Morbidity*

\begin{tabular}{|c|c|c|c|}
\hline & $\begin{array}{l}\text { No. }(\%) \text { in Patients With Severe } \\
\text { Maternal Morbidity, } n=332\end{array}$ & $\begin{array}{l}\text { No. (\%) in Patients Without Severe } \\
\text { Maternal Morbidity, } n=115,156\end{array}$ & $\boldsymbol{P}^{\dagger}$ \\
\hline \multicolumn{4}{|l|}{ Maternal characteristics } \\
\hline Age, years & & & $<0.001$ \\
\hline$<35$ & $232(69.9)$ & $95914(83.3)$ & \\
\hline$\geq 35$ & $100(30.1)$ & $19242(16.7)$ & \\
\hline Race/ethnicity & & & $<0.001$ \\
\hline Non-Hispanic White & $114(34.3)$ & $51922(45.1)$ & \\
\hline Non-Hispanic Black & $110(33.1)$ & $23761(20.6)$ & \\
\hline Non-Hispanic Asian & $22(6.6)$ & $5976(5.2)$ & \\
\hline Hispanic & $66(19.9)$ & $27224(23.6)$ & \\
\hline Other & $16(4.8)$ & $5067(4.4)$ & \\
\hline Not documented & $4(1.2)$ & $1206(1.1)$ & \\
\hline Body mass index at delivery, $\mathrm{kg} / \mathrm{m}^{2}$ & & & $<0.001$ \\
\hline$<25$ & $44(13.5)$ & $14198(12.6)$ & \\
\hline $25-29.9$ & $102(31.4)$ & $41160(36.5)$ & \\
\hline $30-34.9$ & $79(24.3)$ & $32006(28.4)$ & \\
\hline $35-39.9$ & $44(13.5)$ & $15041(13.3)$ & \\
\hline$\geq 40$ & $56(17.2)$ & $10423(9.2)$ & \\
\hline Cigarette use during pregnancy & $57(17.2)$ & $11308(9.8)$ & $<0.001$ \\
\hline Cocaine or methamphetamine use during pregnancy & $9(2.7)$ & $821(0.7)$ & $<0.001$ \\
\hline Insurance status & & & $<0.001$ \\
\hline Non-government assisted & $159(48.3)$ & $69284(60.7)$ & \\
\hline Government-assisted & $170(51.7)$ & $44949(39.4)$ & \\
\hline Prenatal care & $284(96.6)$ & $107213(97.9)$ & 0.11 \\
\hline Obstetric history & & & $<0.001$ \\
\hline Nulliparous & $123(37.1)$ & $46646(40.5)$ & \\
\hline Prior vaginal delivery only & $92(27.7)$ & $49769(43.2)$ & \\
\hline Prior cesarean only & $43(13.0)$ & $8827(7.7)$ & \\
\hline
\end{tabular}




\begin{tabular}{|c|c|c|c|}
\hline & $\begin{array}{l}\text { No. (\%) in Patients With Severe } \\
\text { Maternal Morbidity, } \mathbf{n = 3 3 2}\end{array}$ & $\begin{array}{l}\text { No. (\%) in Patients Without Severe } \\
\text { Maternal Morbidity, } n=115,156\end{array}$ & $P^{\dagger}$ \\
\hline Prior cesarean and vaginal & $74(22.3)$ & $9885(8.6)$ & \\
\hline Any hypertension & $133(40.1)$ & $13133(11.4)$ & $<0.001$ \\
\hline Diabetes mellitus & & & $<0.001$ \\
\hline None & $265(79.8)$ & $106430(92.5)$ & \\
\hline Gestational & $47(14.2)$ & $6951(6.0)$ & \\
\hline Pregestational & $20(6.0)$ & $1712(1.5)$ & \\
\hline Anticoagulant use during pregnancy & $22(6.6)$ & $895(0.8)$ & $<0.001$ \\
\hline Multiple gestation & $30(9.0)$ & $2785(2.4)$ & $<0.001$ \\
\hline Polyhydramnios & $7(2.1)$ & $933(0.8)$ & 0.02 \\
\hline Oligohydramnios & $15(4.5)$ & $4684(4.1)$ & 0.67 \\
\hline Placenta previa & $20(6.0)$ & $447(0.4)$ & $<0.001$ \\
\hline Placenta accreta & $41(12.4)$ & $116(0.1)$ & $<0.001$ \\
\hline Placental abruption & $24(7.2)$ & $906(0.8)$ & $<0.001$ \\
\hline PROM/preterm PROM & $19(6.0)$ & $5983(5.3)$ & 0.56 \\
\hline GBS status & & & $<0.001$ \\
\hline Negative & $125(37.7)$ & $68792(59.7)$ & \\
\hline Positive & $51(15.4)$ & $24335(21.1)$ & \\
\hline Unknown & $156(47.0)$ & $22029(19.1)$ & \\
\hline \multicolumn{4}{|c|}{ Neonatal characteristics (on maternal level, according to first born in multiple gestations) } \\
\hline Presentation at delivery & & & $<0.001$ \\
\hline Vertex & $272(82.4)$ & $109421(95.3)$ & \\
\hline Breech & $47(14.2)$ & $4796(4.2)$ & \\
\hline Non-breech malpresentation & $11(3.3)$ & $656(0.6)$ & \\
\hline Gestational age at delivery, weeks & & & $<0.001$ \\
\hline $23^{0}-27^{6}$ & $28(8.4)$ & $1055(0.9)$ & \\
\hline $28^{0}-33^{6}$ & $57(17.2)$ & $3508(3.1)$ & \\
\hline $34^{0}-36^{6}$ & $82(24.7)$ & $8871(7.7)$ & \\
\hline $37^{0}-37^{6}$ & $34(10.2)$ & $10395(9.0)$ & \\
\hline $38^{0}-38^{6}$ & $32(9.6)$ & 20311 (17.6) & \\
\hline
\end{tabular}




\begin{tabular}{|c|c|c|c|}
\hline & $\begin{array}{r}\text { No. (\%) in Patients With Severe } \\
\text { Maternal Morbidity, } n=332\end{array}$ & $\begin{array}{r}\text { No. }(\%) \text { in Patients Without Severe } \\
\text { Maternal Morbidity, } n=115,156\end{array}$ & $P^{\dagger}$ \\
\hline $39^{0}-39^{6}$ & $52(15.7)$ & $37553(32.6)$ & \\
\hline $40^{0}-40^{6}$ & $30(9.0)$ & $23832(20.7)$ & \\
\hline $41^{0}-41^{6}$ & $16(4.8)$ & $8981(7.8)$ & \\
\hline$\geq 42^{0}$ & $1(0.3)$ & $651(0.6)$ & \\
\hline Size for gestational age & & & $<0.001$ \\
\hline Small & $53(16.0)$ & $10624(9.2)$ & \\
\hline Appropriate & $249(75.2)$ & $95466(82.9)$ & \\
\hline Large & $29(8.8)$ & $9048(7.9)$ & \\
\hline
\end{tabular}

PROM, premature rupture of membranes; GBS, group B streptococcus.

* Severe maternal morbidity defined as a score of at least 8 based on the system of Geller et al. 4

${ }^{\dagger}$ Based on the chi-square or Fisher's exact test.

${ }^{*}$ Race or ethnicity as reported in the chart. 
Table 3

Adjusted Odds Ratios and 95\% Confidence Intervals of Patient Characteristics Associated With Severe Maternal Morbidity*

\begin{tabular}{|c|c|c|c|c|}
\hline Patient Characteristic & Adjusted Odds Ratio & \multicolumn{2}{|c|}{$95 \% \mathrm{CI}$} & Risk Score Points ${ }^{\dagger}$ \\
\hline Age $\geq 35$ years & 1.70 & 1.31 & 2.21 & 2 \\
\hline Cigarette use during pregnancy & 1.44 & 1.06 & 1.96 & 1 \\
\hline Government-assisted insurance & 1.56 & 1.23 & 1.98 & 1 \\
\hline \multicolumn{5}{|c|}{ Obstetric history (referent: prior vaginal delivery only) } \\
\hline Nulliparous & 1.38 & 1.04 & 1.83 & 1 \\
\hline Prior cesarean only & 1.99 & 1.35 & 2.95 & 2 \\
\hline Prior cesarean and vaginal & 2.12 & 1.52 & 2.95 & 2 \\
\hline Any hypertension & 3.36 & 2.61 & 4.32 & 4 \\
\hline \multicolumn{5}{|c|}{ Diabetes mellitus (referent: no diabetes mellitus) } \\
\hline Gestational & 2.07 & 1.49 & 2.88 & 2 \\
\hline Pregestational & 1.63 & 1.00 & 2.64 & 2 \\
\hline Antenatal anticoagulant use & 5.21 & 3.27 & 8.32 & 5 \\
\hline Placenta accreta & 58.11 & 36.84 & 91.64 & 13 \\
\hline Placental abruption & 1.92 & 1.14 & 3.24 & 2 \\
\hline \multicolumn{5}{|c|}{ Gestational age at delivery (referent: $39^{0}-39^{6}$ weeks) } \\
\hline $23^{0}-27^{6}$ & 9.10 & 5.52 & 15.02 & 7 \\
\hline $28^{0}-33^{6}$ & 4.66 & 3.08 & 7.06 & 5 \\
\hline $34^{0}-36^{6}$ & 3.15 & 2.17 & 4.58 & 4 \\
\hline $37^{0}-37^{6}$ & 1.52 & 0.97 & 2.36 & 1 \\
\hline $38^{0}-38^{6}$ & 0.97 & 0.62 & 1.51 & 0 \\
\hline $40^{0}-40^{6}$ & 1.09 & 0.69 & 1.72 & 0 \\
\hline $41^{0}-41^{6}$ & 1.60 & 0.90 & 2.82 & 1 \\
\hline$\geq 42^{0}$ & 1.41 & 0.19 & 10.21 & 1 \\
\hline
\end{tabular}

CI, confidence interval.

Severe maternal morbidity defined as a score of at least 8 based on the system of Geller et al. ${ }^{4}$

${ }^{\dagger}$ Based on dividing each beta by 0.322 , the smallest significant beta (corresponds to nulliparous), and rounding to the nearest integer. 


\begin{tabular}{|c|c|c|c|c|c|c|c|c|c|c|c|c|c|c|c|c|c|c|c|c|c|}
\hline 80 & \begin{tabular}{|c|}
$\mid \widehat{T}$ \\
0 \\
0 \\
0 \\
0 \\
$\dot{e}$ \\
$\underline{y}$ \\
0 \\
0
\end{tabular} & 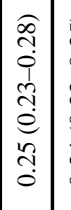 & 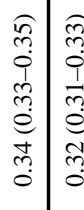 & 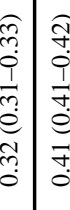 & 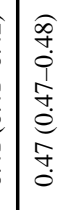 & 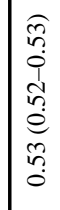 & $\mid$\begin{tabular}{|l}
0 \\
0 \\
0 \\
0 \\
0 \\
$\hat{n}$ \\
0 \\
0 \\
0 \\
0 \\
0
\end{tabular} & 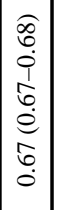 & \begin{tabular}{c|}
$\hat{\hat{N}}$ \\
$\hat{0}$ \\
$\hat{0}$ \\
$\hat{e}$ \\
$\hat{\kappa}$ \\
$\hat{0}$
\end{tabular} & 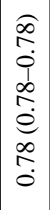 & 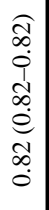 & 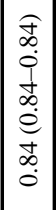 & $\begin{array}{l}0 \\
0 \\
\infty \\
0 \\
1 \\
0 \\
\infty \\
e \\
0 \\
0 \\
\infty\end{array}$ & \begin{tabular}{|c|c}
$\mathscr{\infty}$ \\
$\infty$ \\
0 \\
$\infty$ \\
$\infty$ \\
$\infty$ \\
$e$ \\
$\infty$ \\
$\infty$ \\
$\infty$ \\
0
\end{tabular} & $\begin{array}{l}\hat{\alpha} \\
0 \\
0 \\
o \\
\infty \\
0 \\
0 \\
\infty \\
0\end{array}$ & 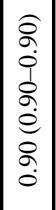 & $\begin{array}{l}\widehat{\sigma} \\
\hat{\sigma} \\
\hat{1} \\
\alpha \\
\hat{\sigma} \\
\hat{\sigma} \\
\sigma \\
\alpha\end{array}$ & $\mid \begin{array}{l}\widehat{\sigma} \\
\hat{0} \\
\hat{\sigma} \\
\hat{\sigma} \\
\hat{\sigma} \\
\sigma\end{array}$ & 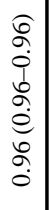 & $\begin{array}{l}0 \\
\circ \\
0 \\
0 \\
\infty \\
o \\
e \\
\infty \\
o \\
0\end{array}$ & $\begin{array}{l}\widehat{\partial} \\
\hat{o} \\
\hat{o} \\
\hat{\sigma} \\
\hat{o} \\
\hat{\sigma} \\
\hat{o}\end{array}$ \\
\hline
\end{tabular}

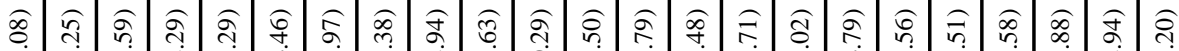

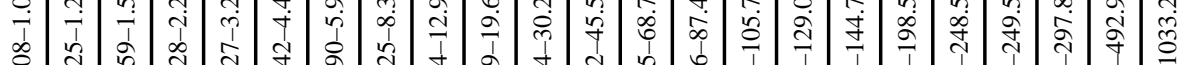

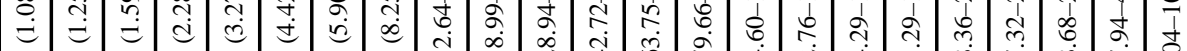

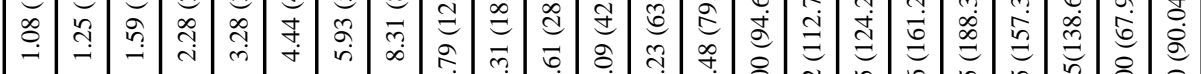

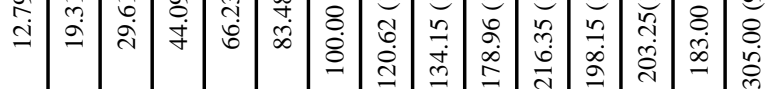

क人

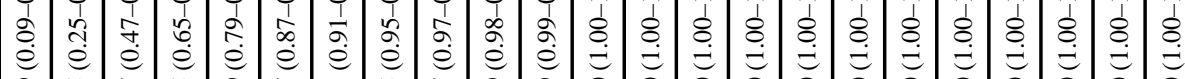

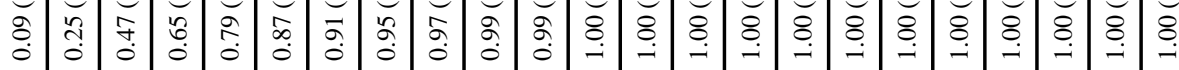

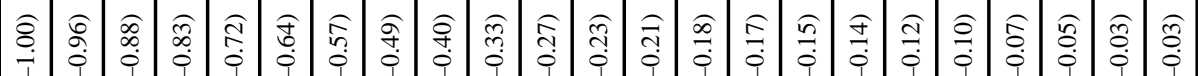

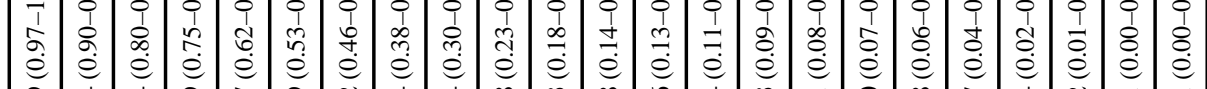

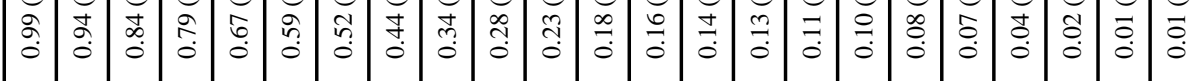

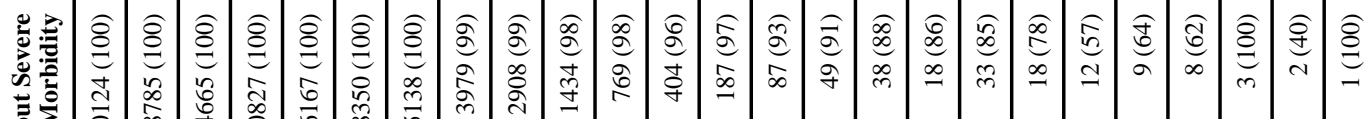

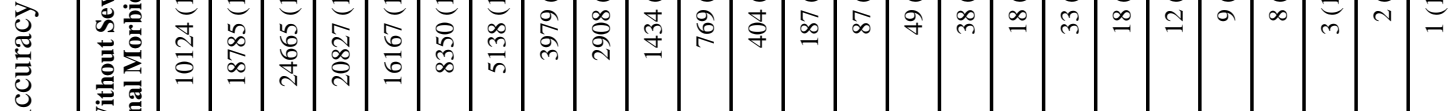

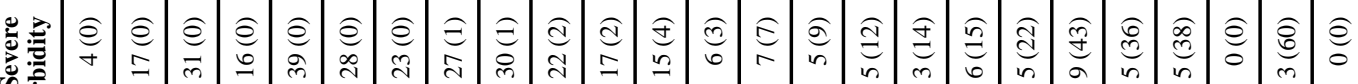

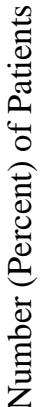

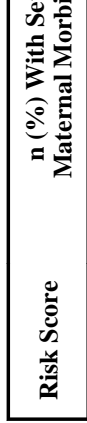

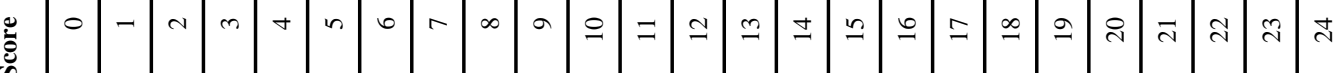
Obstet Gynecol. Author manuscript; available in PMC 2015 April 01. 